\title{
Endoscopic Transnasal Resection of Adenoid Cystic Carcinoma of the Nasal Septum: A Case Report and Literature Review
}

Tessei Kuruma*, Tetsuya Ogawa, Mariko Arimoto, Mayuko Kishimoto, Kinga You, Yuka Kawade, Yutaka Kondo, Yasue Uchida and Yasushi Fujimoto

Department of Otorhinolaryngology, Head and Neck Surgery, Aichi Medical University, Aichi, Japan

*Corresponding author: Tessei Kuruma, Department of Otorhinolaryngology, Head and Neck Surgery, Aichi Medical University, 1-1 Yazakokarimata, Nagakute-shi, Aichi 480-1195, Japan.

Received Date: June 07, 2021

Published Date: June 21, 2021

\section{Abstract}

Very few reports have described adenoid cystic carcinoma of the nasal septum. We performed endoscopic endonasal surgical resection of an adenoid cystic carcinoma of the nasal septum in a 61-year-old woman. Histological examination confirmed adenoid cystic carcinoma. The patient has had no local recurrence or distant metastases as of 3 years postoperatively.

Keywords: Adenoid cystic carcinoma; Nasal septum; Endoscopic endonasal surgery; 4-Hand operation

Abbreviations: Adenoid cystic carcinoma (ACC); Computed tomography (CT); Magnetic resonance imaging (MRI); Positron emission tomography (PET)

\section{Introduction}

Adenoid cystic carcinoma (ACC) is the most common tumor of salivary gland tissues in the head and neck area and the second most common tumor in the nose and sinuses [1,2]. Sinonasal ACC accounts for $10-25 \%$ of all head and neck ACCs [3]. However, this tumor rarely develops in the nasal septum. In fact, very few cases of ACC in the nasal septum have been reported to date [4-6]. Sinonasal tumors are often asymptomatic and are frequently associated with inflammatory disease, leading to delayed diagnosis. The clinical features of ACC differ from those of squamous cell carcinoma, and the features must be recognized for diagnosis and treatment. We report a case of ACC that occurred above the anterior septum.

\section{Case Presentation}

A 61-year-old woman presented to a local otolaryngologist with a 1-year history of right epistaxis. A mass was identified in the right nasal cavity and she was referred to our department for further examination. Nasal endoscopic findings on the first visit revealed a reddish mass that had developed among superficial blood vessels occupying the right nasal cavity (Figure 1). The tumor readily bled simply by inserting gauze into the nasal cavity under local anesthesia for biopsy. Judging from the surface properties of the tumor, it was considered to show abundant blood flow.

Sinus Computed Tomography (CT) showed a $38 \times 15 \mathrm{~mm}$, welldefined, smooth mass shadow in the right middle nasal meatus. The mass excluded the nasal septum but showed no infiltration (Figure 2a). In sinus magnetic resonance imaging (MRI), T2-weighted image, a space-occupying lesion was found in the middle of the right nasal meatus, appearing signal isointense to the mucosa of the nasal cavity. The tumor was in wide contact with the inferior turbinate, but the boundary was clear (Figure $2 b, c$ ). The patient had 
a history of asthma and was unable to undergo diagnostic imaging using contrast media. However, because the mass was considered to have a rich blood supply, we consulted with a radiologist about the utility of angiography. We decided to perform angiography while paying close attention to potential allergies to contrast media. Angiography was performed from the internal carotid artery and external carotid artery, but no deep tumor staining was observed
(Figure 3a,b). From this result, vascular tumors were ruled out. Biopsy of the tumor was performed again at an outpatient clinic. At the time of tumor biopsy, the tumor was squeezed outward with a detacher, and it was found that the tumor originated from the nasal septum. The biopsy was performed under a rigid microscope with an assistant sucking blood from the biopsy site. As a result, bipolar ablation and bleeding could be stopped in a short time.

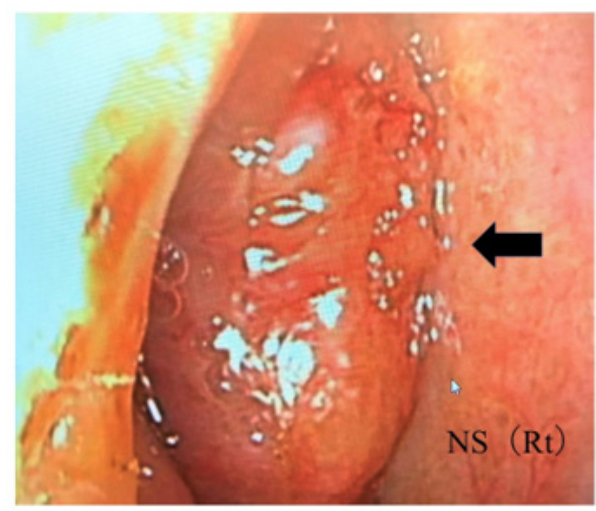

Nasal endoscopic findings show a reddish mass that has developed among the superficial blood vessels occupying the right nasal cavity (arrow). NS: nasal septum.

Figure 1: Nasal Endoscopy.
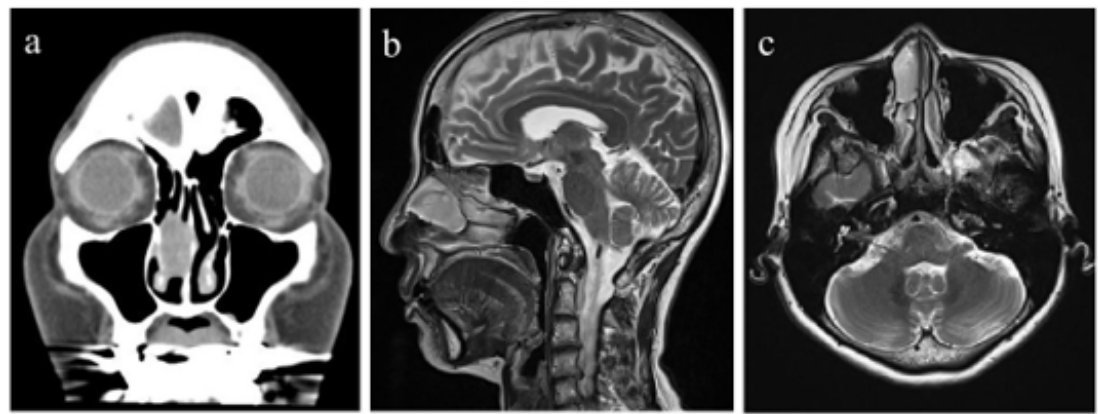

a) Plain computed tomography (CT) of the paranasal sinuses reveals a $38 \times 15-\mathrm{mm}$, well-defined, smooth mass shadow in the right middle nasal meatus.

b) Sagittal T2-weighted MRI shows the mass located anterosuperior to the nasal septum.

c) Axial T2-weighted MRI shows a space-occupying lesion in the right middle nasal meatus with signal isointensity to the mucosa of the nasal cavity.

Figure 2: Preoperative Imaging.
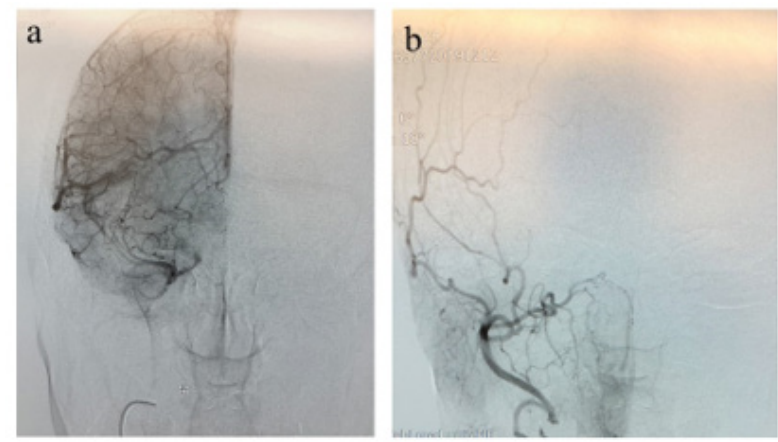

a) Right internal carotid angiography does not show strong staining of the tumor.

b) Right external carotid angiography does not show strong staining of the tumor.

Figure 3: Preoperative angiography. 
Whole-body positron emission tomography (PET)/CT showed no integrated images of cervical and distant metastases other than the nasal cavity. Right nasal ACC (T1N0M0) was diagnosed. The surgery was performed endoscopically under general anesthesia. Since the tumor was located in front of and above the nasal septum, the nasal wing was lifted with a hook to improve the field of view of the endoscope.

The mucosa of the nasal septum above the tumor was confirmed endoscopically, and an arc was incised upward from the nasal septum above the tumor, then the lacrimal bone and anterior surface of the inferior concha were resected with an electric knife (Figure 4a). In addition, the nasal septal mucosa and nasal septal cartilage were incised while maintaining a sufficient safety margin in front of the tumor, and the contralateral nasal septal perichondrium was peeled off. When the inferior and posterior nasal septum mucosa had been sufficiently exfoliated, the nasal septum and lamina perpendicularis of the ethmoid bone were exfoliated, and excision was performed from the opposite side toward the tumor-bearing surface with a safety margin (Figure $4 \mathrm{~b}$ ). The tumor was removed from the olfactory fissure site together with part of the mucosa of the lacrimal bone and middle and inferior conchae (Figure 4c). Rapid intraoperative pathology confirmed the absence of residual tumor, especially in the upper mucosa.
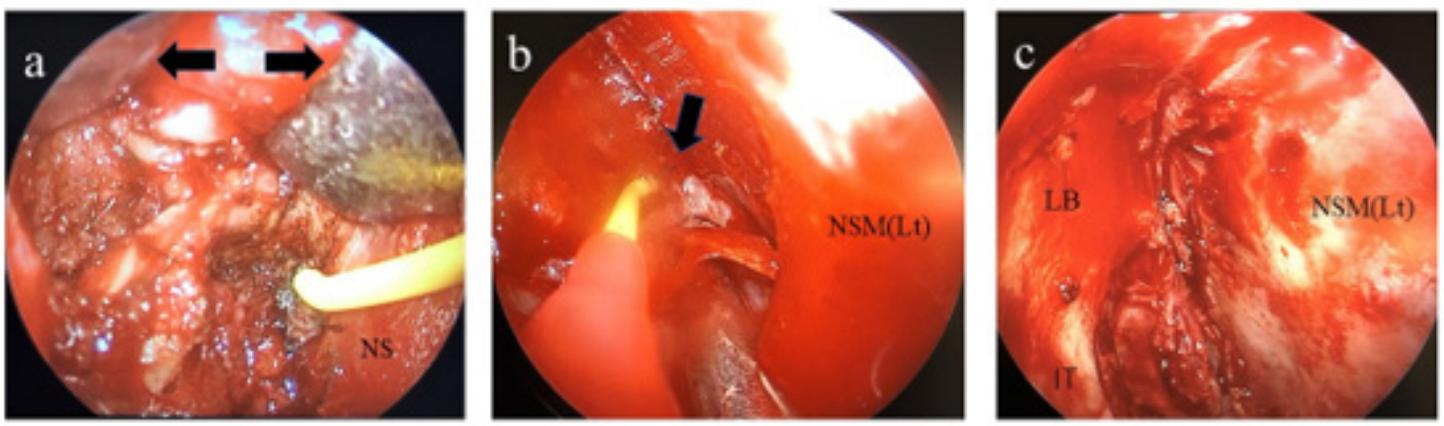

a) While lifting the nasal wing upward with two muscular hooks (arrow), the back part of the nasal wing is excised with an electric knife in front of the tumor. NS: nasal septum side.

b) The contralateral nasal septum is exfoliated and the mucosa is excised above the nasal septum from the opposite side (arrow). NSM(Lt): left nasal septal mucosa.

c) Findings after tumor removal. The mucosa has been extensively resected from above the nasal septum to the lacrimal bone and inferior turbinate. NSM(Lt): left nasal septal mucosa; LB: lacrimal bone; MT: inferior turbinate.

Figure 4: Intraoperative Findings.

Postoperative pathological examination revealed cribriformtype ACC. The tumor was $30 \times 14 \mathrm{~mm}$ in size and showed a biphasic structure of myoepithelial cells and glandular epithelial cells, forming a glandular cavity and growing in a cribriform pattern (Figure 5a). The tumor was found to be infiltrating the nasal septal cartilage but showed no clear perineural or vascular infiltration. The margins and depths of the resected tumor were negative. Based on the results of pathological examination and consultation at a conference with a radiologist, postoperative radiotherapy was considered unnecessary (Figure 5b).
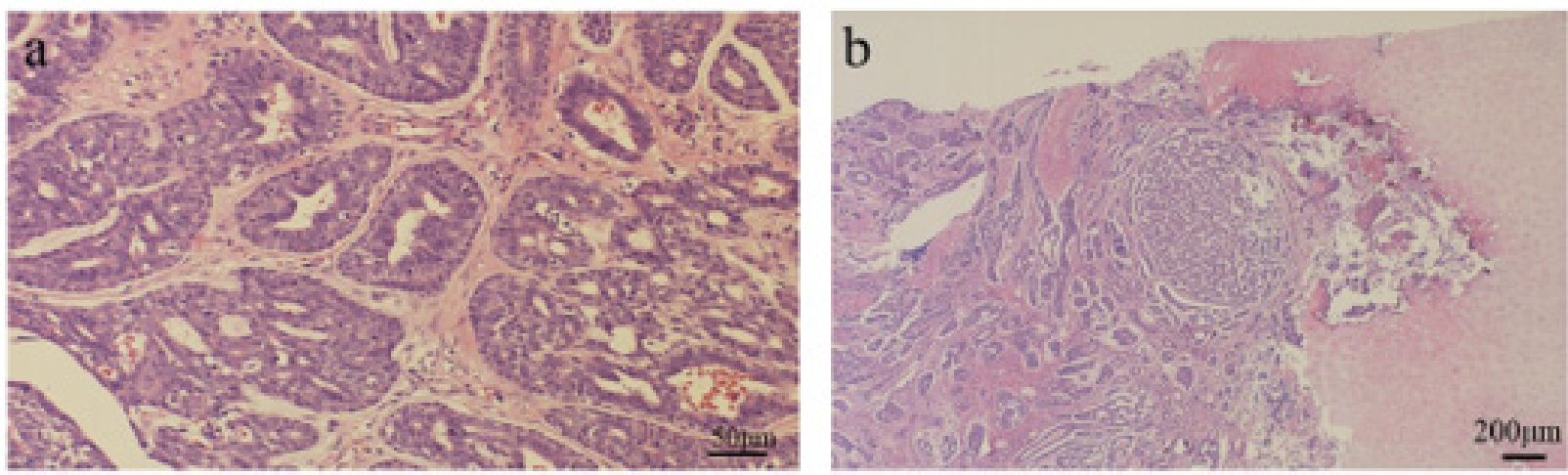

a) Histopathological examination reveals a cribriform variant of adenoid cystic carcinoma (hematoxylin and eosin, $\times 400$ ).

b) Histopathological examination reveals tumor infiltrating the nasal septal cartilage (hematoxylin and eosin, $\times 80$ ).

Figure 5: Histochemical Staining 
Local findings in the nose were observed every month for 1 year after surgery, and were evaluated by CT of the neck, chest and abdomen every 6 months to thoroughly check for local recurrences and distant metastases. From 1 year postoperatively, medical examinations were performed once every 2 months. As of 3 years since the surgery, the patient has shown no local recurrences or metastases of the tumor.

\section{Discussion}

ACC is of epithelial origin and shows a high frequency of local recurrence and distant hematogenous metastatic spread to the lungs, bone, and liver, although lymphatic spread is rare [7]. ACC is the second most common tumor in the nasal cavity and paranasal sinuses after squamous cell carcinoma $[1,8]$. ACC in the nasal cavity and paranasal sinuses origin often shows a worse prognosis than those in other areas of the head and neck. However, nasal cavity tumors have been found to have better outcomes than sites in the paranasal sinus.

ACC is most commonly an asymptomatic, slowly expanding mass, often found in the head and neck [6]. However, nasal ACC may manifest as nasal congestion, epistaxis, secretions, and facial swelling $[9,10,4]$. Pain and paresthesia may also be reported, potentially reflecting a tendency of the tumor toward perineural infiltration [6]. ACC originating from the sinuses is generally asymptomatic and can often be found in a fairly advanced state. However, as in the present case, nasal septal ACC may be more likely to be detected early because symptoms may appear early in the disease course [10].

Local findings in the nasal cavity alone do not allow easy determination of the base and extent of tumor extension, and diagnostic imaging with CT and MRI is very helpful in assessing tumor characteristics and enlargement. In particular, when selecting a surgical method (endoscopic surgery, skull base surgery or removal by external incision), the presence of tumor infiltration along the cranial nerves extending to the skull base is an important consideration. Low-grade ACC in the paranasal sinuses may mimic the characteristics of simple polyps that fill the nasal cavity and show slight bone remodeling on both CT and MRI, while high-grade ACC can manifest as a large, irregular mass with bone destruction on both CT and MRI [11]. Contrast-enhanced imaging is necessary to confirm tumor infiltration into the skull along the cranial nerves and to clarify whether the tumor has abundant blood flow. In the present case, contrast-enhanced MRI could not be performed due to bronchial asthma. However, angiography completely ruled out vascular tumors, and preoperative biopsy was able to be performed safely.

Tissue biopsy is required for definitive diagnosis. However, malignant tumors such as this tumor often bleed. When a tumor develops in the nasal cavity, sinusitis often occurs as a secondary effect, so bleeding from the nasal mucosa is also increased. Since the nasal cavity is narrow, firm contraction of the inside of the nasal cavity with adrenaline gauze is necessary, along with confirmation of the site for the base of the tumor. Furthermore, properties of the tumor surface need to be confirmed under endoscopy and a solid sample must be obtained from the tumor body. In outpatient biopsy, profuse bleeding may prevent collection of sufficient samples. Biopsy can be completed within a short time by cauterizing and stopping bleeding while having an assistant perform blood suction.

Perzin, et al. [12] examined 62 cases of salivary gland ACC. Histologically, they classified three growth patterns: solid, cribriform, and tubular. The cribriform pattern is the most common histological subtype [12]. All nasal septal ACCs reported so far, including this case, have been this type $[5,6,13]$. Perzin et al. [12] also examined tissue type and prognosis. Five-year recurrence rates for solid, cribriform, and tubular-pattern tumors have been reported as $100 \%, 89 \%$, and $59 \%$, with average survivals of 5,8 , and 9 years, respectively [1]. Patients with solid ACC display the worst prognosis [12].

Conventionally, among the histological types of adenoid cystic carcinoma, the tubular type has been considered to represent the most differentiated type, and the solid type has been considered to represent the least differentiated type $[12,14]$. Yamamoto, et al. [15]. suggested that ACC changes from well-differentiated to poorly differentiated over time, resulting in increased tumor growth potential and the possibility of recurrence and metastasis [15]. Szanto, et al. [16] subdivided ACC into three groups: Grade 1, solid pattern 0\%; Grade 2, solid pattern $>0 \%$ but $<30 \%$, and Grade 3, solid pattern $\geq 30 \%$, depending on the mix of ACC tissue types. They reported 5-year survival rates of $92 \%, 65 \%$, and $14 \%$, respectively, and 10 -year survival rates of $76 \%, 26 \%$, and $5 \%$, respectively. A detailed pathological assessment of the proportion of solid pattern thus has important prognostic value in ACC [16].

Surgical excision is the modality of choice for the treatment of nasal septal malignancies. The surgical approach is modified for the individual tumor according to the size and location of the tumor. Recently, some authors have deemed surgical resection of ACC of the sinonasal tract via an endoscopic approach as an effective, minimally invasive method that in well-selected patients could achieve satisfactory application, and together with adjuvant therapies providing good results, complete removal of the tumor and a high survival rate [17].

Surgery for malignant tumor of the nasal septum requires consideration of the most suitable method for removing the tumor with a sufficient safety margin. Endoscopic surgery is superior to external incision and intracranial surgery in terms of esthetic outcomes and side effects. However, limits exist to the indications for endoscopic surgery. According to Stammberger, et al. [18] the limitations of the endoscopic technique result from the anatomical spread of the tumor, when there is extensive infiltration of the orbit, 
dura/brain, and other vital structures [18]. The key anatomical structures include the internal carotid artery, optic nerve, cavernous sinuses, and ethmoid roof [19].

Most researchers reserve the endonasal method for experienced surgeons and consider the possibility of its application to depend on TNM staging [18]. According to Hanna, et al. [20] endoscopically treated tumors are usually of lower stage, i.e., T1T2 [20]. They emphasized the necessity of cooperation between the endorhinolaryngologist and neurosurgeon [18]. Our hospital has some specialists in skull base surgery and ocular plastic surgery, so we consult with them regarding tumors near the orbit or skull base and decide on the surgical method without sticking to endoscopic surgery. The combined use of an endoscope is also useful for external incision and intracranial surgery. Substantial developments in endoscopy have been made in recent years, and improvements in image quality have made it possible to ensure that endoscopy provides a much safer margin than tumor removal under the naked eye.

In endoscopic surgery, if the field of view deteriorates with bleeding, etc., or if the expectation is that the surgical field cannot be expanded well due to the narrow working space in the nasal cavity, an auxiliary external incision will be required. For anterior nasal septal tumors, lateral rhinotomy is the preferred surgical approach, whereas sublabial incision with Denkers's approach is considered more appropriate for posterior nasal tumors [10].

The primary lesion in this case was early-stage T1. However, because the tumor was well anterior and superior to the septum, the point was whether the upper part of the nasal septum above the tumor and the back surface of the ala of the nose could be clearly seen. The operation was performed by one assistant lifting the nasal wings with a hook and another assistant suctioning blood or cauterizing with an electric scalpel. By performing endoscopic surgery using these 4-5 hands, the area around the tumor was clearly visible. The extent of tumor progression and the surgical procedure could be shared by all surgeons at the preoperative conference.

ACC is considered to be radiosensitive, but not radiocurable, so the rationale for administering postoperative radiation therapy is to treat any residual microscopic disease, which is present in most patients [21]. Postoperative radiation is now generally recommended. However, the benefit of adjuvant radiotherapy has never been clearly demonstrated in the absence of a randomized prospective trial [22-24].

A meta-analysis by Amit, et al. [25] revealed margin status and tumor site as significant predictors of outcome in ACC patients with tumor in the sinuses and skull base, whereas perineural infiltration was not associated with prognosis. As an important result, Amit, et al. [25] identified no additional benefits from adjuvant therapy [25].
As surgery of the primary site was the only demonstrated clinical intervention noted to confer survival benefit on patients diagnosed with ACC in the head and neck region, evidence supports surgical therapy remaining as the mainstay of therapy, at least for now. Therefore, in the treatment of ACC of the nasal septum, removal with a sufficient safety margin is of primary importance. Postoperative radiotherapy is required if the surgical margin of the tumor appears microscopically positive.

\section{Conclusion}

We encountered a 61-year-old woman with ACC of the nasal septum. Endoscopic removal surgery was performed. Since the tumor was located above the anterior septum, 4- or 5-hand endoscopic surgery could be performed and the tumor was removed with a sufficient safety margin, even in the narrow surgical field. Following thorough examination with the radiology department, postoperative radiotherapy was not performed. When performing nasal septal surgery for ACC, the extent of lesion extension should be grasped by local and imaging findings, and a method for tumor removal with a sufficient safety margin should be devised. ACC shows a high incidence of local recurrence or distant metastasis, so careful, long-term follow-up is warranted.

\section{Acknowledgement}

none.

\section{Conflict of Interest}

The authors declare no potential conflicts of interest.

\section{Funding}

The authors received no specific funding for this work.

\section{References}

1. Rhee CS, Won TB, Lee CH, Min YG, Sung MW, et al. (2006) Adenoid cystic carcinoma of the sinonasal tract: treatment results. Laryngoscope 116(6): 982-986

2. Cruz Perez DE, Pires FR, Lopes MA, de Almeida OP, Kowalski LP, et al. (2006) Adenoid cystic carcinoma and mucoepidermoid carcinoma of the maxillary sinus: report of a 44-year experience of 25 cases from a single institution. J Oral Maxillofac Surg 64(11): 1592-1597.

3. Purushothaman PK, Ramakrishnan R, Sindhu P (2015) Adenoid cystic carcinoma of nasal cavity: a rare presentation case report. Int J Sci Res Publ 5(2): 1-3.

4. Schneiderman TA, Chaudhury SI (2002) Adenoid cystic carcinoma of the nasal septum. Otolaryngol Head Neck Surg 127(3): 251-252.

5. Sivaji N, Basavaraj S, Stewart W, Dempster J (2003) Adenoid cystic carcinoma of the nasal septum. Rhinology 41(4): 253-254.

6. Tai SY, Chien CY, Tai CF, Kuo WR, Huang WT, et al. (2007) Nasal septum adenoid cystic carcinoma: a case report. The Kaohsiung Journal of Medical Sciences 23(8): 426-430.

7. Shishodia S, Narayan U, Sharma S (2020) An unusual case of adenoid cystic carcinoma of nasal septum mimicking as chronic sinusitis. Otorhinolaryngol Head Neck Surg 5(1): 1839. ISSN: 2474-1655.

8. Da Crus Perez DE, Pires FE, Lopes MA, de Almeida OP, Kowalski LP, et al. (2006) Adenoid cystic carcinoma and mucoepidermoid carcinoma of the maxillary sinus: report of a 44-year experience of 25 cases from a single institution. J Oral Maxillofac Surg 64(11): 1592-1597. 
9. Dillon PM, Chakraborty S, Moskaluk CA, Joshi PJ, Thomas CY, et al. (2016) Adenoid cystic carcinoma: a review of recent advances, molecular targets, and clinical trials. Head Neck 38(4): 620-627.

10. Akiyama K, Karaki M, Hosikawa H, Mori N (2013) A massive adenoid cystic carcinoma of nasal septum progressed into the skull base. Auris Nasus Larynx 40(2): 239-242.

11. Kouadir A, Hassouni K (2018) Early-stage adenoid cystic carcinoma of the posterior nasal septum: a rare manifestation. NOWOTWORY Journal of Oncology 68 (1): 33-37.

12. Perzin KH, Gullane P, Clairmont AC (1978) Adenoid cystic carcinomas arising in salivary glands: a correlation of histologic features and clinical course. Cancer 42(1): 265-282

13. Handa T, Yamamoto H, Yamakawa J, Yoshihiro T, Hayashi T, et al. (1992) A case report of adenoid cystic carcinoma of the nasal septum. Nihon Jibiinkoka Gakkai Kaiho 95(4): 505-509.

14. Chomette G, Auriol M, Tranbaloc P, Vaillant JM (1982) Adenoid cystic carcinoma of minor salivary glands. Analysis of 86 cases. Clinicopathological, histoenzymological and ultrastructural studies. Virchows Arch A Pathol Anat Histol 395(3): 289-301.

15. Yamamoto Y, Saka T, Takahashi H (1991) Adenoid cystic carcinoma; a review of recent findings. Practica Oto-Rhino-Laryngologica 84: 11531166.

16. Szanto PA, Luna MA, Tortoledo ME, White RA (1984) Histologic grading of adenoid cystic carcinoma of the salivary glands. Cancer 54(6): 10621069.

17. Wardas P, Tymowski M, Piotrowska Seweryn A, Kaspera W, Ślaska Kaspera A, et al. (2015) Endoscopic approach to the resection of adenoid cystic carcinoma of paranasal sinuses and nasal cavity: case report and own experience. Eur J Med Res 20: 97.
18. Stammberger H, Anderhuber W, Walch C, Papaefthymiou G (1999) Possibilities and limitations of endoscopic management of nasal and paranasal sinus malignancies. Acta Otorhinolaryngol Belg 53(3): 199205.

19. Solares CA, Ong YK, Snyderman CH (2010) Transnasal endoscopic skull base surgery: what are the limits? Curr Opin Otolaryngol Head Neck Surg 18(1): 1-7.

20. Hanna E, DeMonte F, Ibrahim S, Roberts D, Levine N, et al. (2009) Endoscopic resection of sinonasal cancers with and without craniotomy: oncologic results. Arch Otolaryngol Head Neck Surg 135(12): 12191224

21. Leafstedt SW, Gaeta JF, Sako K, Marchetta FC, Shedd DP (1971) Adenoid cystic carcinoma of major and minor salivary glands. Am J Surg 122(6): 756-762.

22. Lupinetti AD, Roberts DB, Williams MD, Kupferman ME, Rosenthal DI (2007) Sinonasal adenoid cystic carcinoma: the M. D. Anderson Cancer Center experience. Cancer 110(12): 2726-2731.

23. Iseli TA, Karnell LH, Graham SM, Funk GF, Buatti JM, et al. (2009) Role of radiotherapy in adenoid cystic carcinoma of the head and neck. J Laryngol Otol 123 (10): 1137-1144.

24. Lloyd S, Yu JB, Wilson LD, Decker RH (2011) Determinants and patterns of survival in adenoid cystic carcinoma of the head and neck, including an analysis of adjuvant radiation therapy. Am J Clin Oncol 34(1): 76-81.

25. Amit M, Binenbaum Y, Sharma K, Naomi R, Ilana R, et al. (2013) Adenoid cystic carcinoma of the nasal cavity and paranasal sinuses: a metaanalysis. J Neurol Surg B Skull Base 74(30): 118-125. 